\title{
Global Health Security Implementation: Expanding the Evidence Base
}

Erin D. Kennedy, Juliette Morgan, and Nancy W. Knight

$\mathbf{I}_{\mathrm{p}}^{\mathrm{N}}$ RECENT YEARS, the world has seen a series of alarming public health emergencies across the globe that have resulted in thousands of lives lost and billions of dollars in economic losses. ${ }^{1-8}$ The frequency and impact of these emergencies, coupled with the ease and reach of international travel and rapidly expanding global commerce and trade, have raised the urgency for all countries to prepare for future emergencies. As we write this piece, the second Ebola outbreak in 2018 in the Democratic Republic of the Congo (DRC) is accelerating in a resource-limited environment and armed conflict zone. This outbreak represents another test of the global health community's ability to contain this threat and prevent global spread.'

The severe acute respiratory syndrome (SARS) outbreak in 2002-03 highlighted the need for updated international guidance for cooperation between countries and the need for all countries to develop core capacities to detect, report, and respond to infectious disease threats at the source. ${ }^{10,11}$ The impact of SARS highlighted the importance of improving global health security and accelerated the adoption of the revised International Health Regulations (IHR 2005). By 2007, all 196 World Health Organization (WHO) member states had committed to reaching compliance with these regulations by 2012. ${ }^{12}$ However, more than two-thirds of countries had failed to meet these standards by $2014 .^{13}$

Global deficiencies in preparedness for infectious disease threats were clear during the 2009 influenza $A(H 1 N 1)$ pandemic, the 2014-2016 Ebola epidemics, the global spread of Zika and chikungunya, and multiple other out- breaks. ${ }^{10}$ One factor impeding progress is limited published evidence regarding what specific approaches and interventions are most effective and have the most impact in enhancing a country's capacity for health security. ${ }^{14}$

To address this evidence gap, the US Centers for Disease Control and Prevention (CDC), as a WHO Collaborating Center for the Implementation of IHR Core Capacities, works with its partners to develop the evidence base for effective global health security interventions. At CDC, the efforts to improve global health security implementation are primarily focused on strengthening public health systems to increase the capacity to prevent, detect, and respond to public health threats. These capacities include surveillance, public health laboratory systems, workforce development, outbreak investigation, and emergency response. Through CDC's direct engagement and collaboration with partners, $\mathrm{CDC}$ provides technical, scientific, and fiscal support to a number of countries to implement activities that build toward sustainable global health security systems in countries and regions. ${ }^{15,16}$

This supplement shares the lessons learned from efforts to build global health security capacity in multiple countries spanning Africa, Asia, and Latin America. We grouped the articles in the supplement into 4 sections: Overview, Prevent, Detect, and Response, in alignment with the Global Health Security Agenda action packages. ${ }^{17}$

The Overview section contains articles relevant to the foundational capacities needed to meet compliance with IHR 2005. In this section, Menon et al present 3 case

Erin D. Kennedy, DVM, MS, MPH, is Associate Director for Science, Epidemiology, Informatics, Surveillance, and Laboratory Branch, Division of Global Health Protection, Center for Global Health; Juliette Morgan, MD, was Associate Director for Science, Division of Global Health Protection, Center for Global Health; and Nancy W. Knight, MD, is a captain in the US Public Health Service and Director, Division of Global Health Protection, Center for Global Health; all at the Centers for Disease Control and Prevention, Atlanta, GA. The findings and conclusions in this report are those of the authors and do not necessarily represent the official position of the Centers for Disease Control and Prevention. 
studies to describe a role for law in IHR 2005 implementation. ${ }^{18}$ Liberia is in the process of updating their 1976 public health law to reflect current public health practices and strengthen IHR 2005 implementation. Côte d'Ivoire and Togo are using law to operationalize their public health emergency operations centers in order to respond to outbreaks in a timely and efficient manner. Cambodia is developing a proclamation designed to strengthen laboratory biosafety and biosecurity practices.

This section also includes an article that describes Pakistan's use of the Joint External Evaluation continuum to develop a costed national action plan for health security (NAPHS). ${ }^{19,20}$ The article describes the lessons learned during the process, which may help countries using the process to develop their own NAPHS. Another article in this section describes how the Staged Development Tool (SDT) and process can be used to help national public health institutes (NPHIs) assess their current capacity and develop plans for addressing identified gaps and moving the country closer to achieving their global health security goals. ${ }^{21}$ The Staged Development Tool and process can also be used to determine the effectiveness of the interventions and changes made by NPHIs to address gaps identified during the original assessment.

The Prevent section contains articles describing specific interventions and assessments designed to strengthen a country's ability to prevent avoidable outbreaks and drive health security planning in countries. Weber et al describe a pilot implementation of the WHO and United Nations Children's Fund's Water and Sanitation for Health Facility Improvement Tool (WASH FIT) in Togo. ${ }^{22}$ While using WASH FIT, internal teams regularly perform self-assessments at their facilities using water, sanitation, and hygiene (WASH) and related management indicators to develop and implement an improvement plan. Facilities made improvements without significant external financial or material support, such that the percent of facilities meeting baseline standards increased from $18 \%$ to $44 \%$ after only 7 months. This pilot evaluation suggests that WASH FIT may help facilities improve WASH services and practices. Applying WASH FIT and the implementation lessons learned can contribute to global health security by helping health facilities in Togo and other countries be more prepared to prevent healthcareassociated infections by limiting the spread of infectious diseases, such as Ebola and other diseases of epidemic and pandemic concern.

Antimicrobial resistance (AMR) and zoonotic disease are 2 key action packages that fall within the Prevent priority area. ${ }^{17}$ Another article included in this section describes the establishment of a laboratory-based AMR surveillance system in Ethiopia; lessons learned from implementation of this system can help guide other countries in implementing similar systems. ${ }^{23}$ This section also includes 2 articles focused on prevention and control of zoonotic diseases. Machalaba et al provide a description of how a multisectoral One Health approach can enhance global health security. ${ }^{24}$ An article by Stolka et al describes an assessment of a priority zoonotic disease surveillance system in the DRC. $^{25}$ The articles by Stolka and Weber are examples of the use of implementation science to conduct structured evaluations that can be used to guide future global health security activities.

The Detect section includes articles that describe the implementation and evaluation of surveillance systems that both enhance the ability to detect public health threats early and build laboratory capacity. In this section, Clara et al describe an evaluation of a community event-based surveillance system in Vietnam implemented to improve the early detection of public health threats. ${ }^{26}$ The evaluation showed the importance of supervision and monitoring of healthcare workers; it also emphasized the need to conduct surveillance evaluations to identify implementation barriers.

Lamorde et al describe a cross-cutting approach to building multisectoral surveillance and laboratory capacity in Uganda by leveraging an existing pediatric inpatient malaria surveillance platform to develop an integrated platform. ${ }^{27}$ Development of this integrated platform has improved Uganda's ability to detect pathogens of public health importance by strengthening surveillance, increasing laboratory capacity, and supporting timely submission of testing data to public health authorities.

Another key factor in a country's ability to detect public health threats is the existence of a workforce trained to recognize outbreaks and conduct investigations. Ario et al describe accomplishments of the Uganda Public Health Fellowship Program, an inservice post-master's advanced Field Epidemiology Training Program (FETP) established in $2015 .^{28}$ The program has been fully integrated into the public health emergency response system. As of the end of 2017, FETP advanced fellows had conducted more than 60 outbreak investigations, 12 public health emergency assessments, 40 surveillance projects, and 31 applied epidemiologic studies, of which 49 involved potential bioterrorism agents or epidemic-prone diseases. The investigations conducted by fellows have resulted in shortened time to identify pathogens responsible for outbreaks, thereby preventing the potential spread of these outbreaks. Fellows graduate with the necessary skills and experiences in field epidemiology and public health leadership to make a significant impact on Uganda's ability to prevent, detect, and respond to public health threats.

The Response section includes articles focused on implementation of programs designed to build capacities for rapid and effective responses to public health emergencies. Hanson et al describe the implementation and evaluation of a regional training pilot designed by CDC to build the capacities in the ministries of health in 4 Latin American countries (Colombia, Dominican Republic, Guatemala, and Panama) to design, facilitate, and evaluate simulation exercises. ${ }^{29}$ Six months after the training, $87.5 \%$ of respondents reported that they had applied the concepts learned in their day-to-day jobs. This pilot program helped 
these 4 countries meet the IHR 2005 requirement of having the ability to conduct exercises and enhance their public health emergency preparedness capacity, thus making them better prepared to respond to public health threats. Sanou et al describe implementation and associated costs of enhanced laboratory-based arbovirus surveillance in Burkina Faso during a dengue outbreak. ${ }^{30}$ This work built on existing infrastructure in Burkina Faso to rapidly respond to the outbreak.

The articles in this supplement provide outcomes and lessons learned from the past 4 years of Global Health Security Agenda implementation, which can provide guidance to other countries implementing similar efforts to build capacities to prevent, detect, and respond to public health threats. The findings presented in these articles begin to provide the information needed to fill the lack of evidence base for global health security implementation. ${ }^{14}$

So that we continue to build on the momentum established through investments to date and do not lose ground on the progress made, it is critical that CDC and partners incorporate the implementation science aspect of the scientific framework suggested by Morgan et al into all global health security capacity-building efforts. ${ }^{31}$ The evidence base needed can be generated through the use of implementation science to evaluate new and existing public health programs and systems. Implementation science can provide evidence on the most effective, efficient, feasible, and sustainable implementation approaches to global health security, which can in turn help strengthen existing programs or be used to develop new programs and move countries toward IHR 2005 compliance.

\section{ACKNOWLEDGMENTS}

We would like to thank the authors who have contributed their work to this supplement, the WHO, partner organizations, and the leadership in other countries' governments for their commitment to IHR 2005 and achieving a world that is safer from public health threats. We also thank Keisha Bohannon, Zara Ahmed, Ruth Cooke Gibbs, Jessica Gershick, Diane Brodalski, and Becky Bunnell for their assistance with this commentary.

\section{REFERENCES}

1. Peiris JS, Yuen KY, Osterhaus AD, Stöhr K. The severe acute respiratory syndrome. $N$ Engl J Med 2003;349(25):24312441. doi:10.1056/NEJMra032498.

2. Fineberg HV. Pandemic preparedness and response-lessons from the H1N1 influenza of 2009. N Engl J Med 2014; 370(14):1335-1342. doi: 10.1056/NEJMra1208802

3. Assiri A, McGeer A, Perl TM, et al.; KSA MERS-CoV Investigation Team. Hospital outbreak of Middle East respiratory syndrome coronavirus. N Engl J Med 2013;369(5):407-416. doi: 10.1056/NEJMoa1306742.
4. Bell DM, Damon I, Bedrosian SR, Johnson VR, McQuiston JH, O'Connor J. CDC's response to the 2014-2016 Ebola epidemic-West Africa and United States. MMWR Suppl 2016;65(3):1-106. https://www.cdc.gov/mmwr/volumes/65/ su/pdfs/su6503.pdf. Accessed November 7, 2018.

5. Ikejezie J, Shapiro CN, Kim J, et al. Zika virus transmission-region of the Americas, May 15, 2015-December 15, 2016. MMWR Morb Mortal Wkly Rep 2017;66(12):329-334. doi: dx.doi.org/10.15585/mmwr.mm6612a4.

6. Cassell CH, Bambery Z, Roy K, et al. Relevance of global health security to the US export economy. Health Secur 2017;15(6):563-568. doi: 10.1089/hs.2017.0051.

7. The World Bank. The Economic Impact of the 2014 Ebola Epidemic: Short and Medium Term Estimates for West Africa. 2014. http://documents. Worldbank.org/curated/en/ 5245214 68141287875/. Accessed November 8, 2018.

8. Bambery Z, Cassell CH, Bunnell RE, et al. Impact of a hypothetical infectious disease outbreak on US exports and export-based jobs. Health Secur 2018;16(1):1-7. doi: 10.1089/ hs.2017.0052.

9. Nakkazi E. DR Congo Ebola virus outbreak: responding in a conflict zone. Lancet 2018;392(10148):623. doi: 10.1016/ S0140-6736(18)31981-0.

10. Tappero JW, Cassell CH, Bunnell RE, et al.; Global Health Security Science Group. US Centers for Disease Control and Prevention and its partners' contributions to global health security. Emerg Infect Dis 2017;23(Suppl 1). doi: 10.3201/ eid2313.170946.

11. National Academies of Sciences, Engineering, and Medicine; Health and Medicine Division; Board on Global Health; Committee on Global Health and the Future of the United States. Global Health and the Future Role of the United States. Washington, DC: National Academies Press; 2017.

12. Rodier G, Greenspan AL, Hughes JM, Heymann DL. Global public health security. Emerg Infect Dis 2007;13(10): 1447-1452. doi: 10.3201/eid1013.070732.

13. Gostin LO, Katz R. The International Health Regulations: the governing framework for global health security. Milbank Q 2016;94(2):264-313.

14. Angulo FJ, Cassell CH, Tappero JW, Bunnell RE. Progress and opportunities for strengthening global health security. Emerg Infect Dis 2017;23(Suppl 1):S1-S4. doi:10.3201/ eid2313.171758.

15. Fitzmaurice AG, Mahar M, Moriarty LF, et al.; GHSA Implementation Group. Contributions of the US Centers for Disease Control and Prevention in implementing the Global Health Security Agenda in 17 partner countries. Emerg Infect Dis 2017;23(Suppl 1). doi: 10.3201/eid2313. 170898.

16. Shoemaker TR, Balinandi S, Tumusiime A, et al. Impact of enhanced viral haemorrhagic fever surveillance on outbreak detection and response in Uganda. Lancet Infect Dis 2018; 18(4):373-375. doi: 10.1016/S1473-3099(18)30164-6.

17. US Centers for Disease Control and Prevention. Global Health Security Agenda: action packages CDC website. Updated January 21, 2016. https://www.cdc.gov/globalhealth/ security/actionpackages/default.htm. Accessed November 8, 2018.

18. Menon AN, Rosenfield E, Brush CA. Law and the JEE: lessons for IHR implementation. Health Secur 2018; 16(Suppl 1):S11-S17. 
19. Barzilay EJ, Vandi H, Binder S, et al. Use of the staged development tool for assessing, planning, and measuring progress in the development of national public health institutes. Health Secur 2018;16(Suppl 1):S18-S24.

20. Bell E, Tappero JW, Ijaz K, et al. Joint external evaluationdevelopment and scale-up of global multisectoral health capacity evaluation process. Emerg Infect Dis 2017;23(Suppl 1). doi: 10.3201/eid2313.170949.

21. Safi M, Ijaz K, Samhouri D, et al. Development of a costed national action plan for health security in Pakistan: lessons learned. Health Secur 2018;16(Suppl 1):S25-S29.

22. Weber N, Martinsen AL, Sani A, et al. Strengthening healthcare facilities through water, sanitation, and hygiene (WASH) improvements: a pilot evaluation of "WASH FIT" in Togo. Health Secur 2018;16(Suppl 1):S54-S65.

23. Hazim C, Ibrahim RA, Westercamp M, et al. Establishment of a sentinel laboratory-based antimicrobial resistance surveillance network in Ethiopia. Health Secur 2018; 16(Suppl 1):S30-S36.

24. Machalaba CC, Salerno RH, Behravesh CB, et al. Institutionalizing One Health: from assessment to action. Health Secur 2018;16(Suppl 1):S37-S43.

25. Stolka KB, Ngoyi BF, Grimes KEL, et al. Assessing the surveillance system for priority zoonotic diseases in the Dem- ocratic Republic of the Congo, 2017. Health Secur 2018; 16(Suppl 1):S44-S53.

26. Clara A, Dao ATP, Do TT, et al. Factors influencing community event-based surveillance: lessons learned from pilot implementation in Vietnam. Health Secur 2018;16(Suppl 1):S66-S75.

27. Lamorde M, Mpimbaza A, Walwema R, et al. A cross-cutting approach to surveillance and laboratory capacity as a platform to improve health security in Uganda. Health Secur 2018;16(Suppl 1):S76-S86.

28. Ario AR, Wanyenze RK, Opio A, et al. Strengthening global health security through Africa's first absolute post-master's fellowship program in field epidemiology in Uganda. Health Secur 2018;16(Suppl 1):S87-S97.

29. Hanson K, Hernandez L, Banaski J Jr. Building simulation exercise capacity in Latin America to manage public health emergencies. Health Secur 2018;16(Suppl 1):S98-S102.

30. Sanou AS, Dirlikov E, Sondo KA, et al. Building laboratorybased arbovirus sentinel surveillance capacity during an ongoing dengue outbreak, Burkina Faso, 2017. Health Secur 2018;16(Suppl 1):S103-S110.

31. Morgan J, Kennedy ED, Pesik N, Angulo FJ, Craig AS, Knight NW, Bunnell RE. Building global health security capacity: the role for implementation science. Health Secur 2018;16(Suppl 1):S5-S7. 